\title{
The Clinical Value of Klotho and FGF23 in Cardiac Valve Calcification Among Patients with Chronic Kidney Disease
}

This article was published in the following Dove Press journal: International Journal of General Medicine

\section{Yan Chen \\ Yan-Xia Chen \\ Chong Huang \\ Zhi-Bing Duan \\ Cheng-Yun Xu}

Department of Nephrology, The Second Affiliated Hospital of Nanchang

University, Nanchang, Jiangxi Province,

330006, People's Republic of China
Correspondence: Yan Chen

Department of Nephrology, The Second Affiliated Hospital of Nanchang

University, No. I Minde Road, Donghu

District, Nanchang, Jiangxi Province,

330006, People's Republic of China

$\mathrm{Tel} / \mathrm{Fax}+8679186312319$

Email chenyan_drcy@163.com
Objective: This study aims to investigate the clinical value of serum Klotho and FGF23 in cardiac valve calcification in patients with chronic kidney disease (CKD).

Methods: In the present study, 180 patients with CKD, who were admitted to the department of nephrology of our hospital on April 1, 2016 (solstice, 2019), were selected as the main subjects. According to the CKD stage, these patients were divided into three groups: CKD2 3 group, CKD4 group, and CKD5 group. In each group, ultrasound was used to evaluate the cardiac valve calcification, and the independent risk factors for cardiac valve calcification were analyzed by Logistic regression.

Results: The levels of hemoglobin and blood calcium in CKD2 3 patients were higher than those in CKD4 and CKD5 patients, and the levels of hemoglobin and blood calcium in CKD5 patients were higher than those in CKD4 patients $(P<0.05)$. Albumin was lower in CKD2 3 patients when compared to CKD5 patients while albumin was higher in CKD5 patients when compared to CKD4 patients $(P<0.05)$. The serum levels of FGF23 was lower in CKD2 3 patients when compared to CKD4 and CKD5 patients while the serum levels of FGF23 was lower in CKD4 patients when compared to CKD5 patients $(P<0.05)$. The serum levels of Klotho was higher in CKD2 3 patients, when compared to CKD4 and CKD5 patients, while the serum levels of Klotho was higher in CKD4 patients, when compared to CKD5 patients $(P<0.05)$. The logistic regression analysis revealed that GFR, serum creatinine, FGF23 and Klotho were independent risk factors for cardiac valve calcification in patients with CKD.

Conclusion: With the decrease of GFR in CKD patients, the serum levels of FGF23 increases, while the serum levels of Klotho decreases. Furthermore, the serum levels of FGF23 and Klotho are affected by various factors, and the levels of FGF23 and Klotho in CKD patients are negatively correlated. GFR, serum creatinine, FGF23 and Klotho are independent risk factors for heart valve calcification in patients with CKD.

Keywords: Klotho, FGF23, chronic kidney disease, cardiac valve calcification, logistic regression analysis

\section{Introduction}

Chronic kidney disease (CKD) increases the risk of developing end-stage renal disease (ESRD), and hypertension and albuminuria have been identified as risk factors for disease progression. ${ }^{1-3}$ The calcification of large arteries and heart valves is common in CKD patients, and may lead to a significant increase in cardiovascular risk. ${ }^{4-7}$ Abdominal aortic calcification is an independent risk factor for cardiovascular events in non-dialysis patients with CKD. ${ }^{8,9}$ Valve calcification 
has been deemed to be closely associated with cardiovascular events and all-cause mortality in patients with CKD. ${ }^{10,11}$ Since valve calcification is strongly associated with cardiovascular events and all-cause mortality in patients with CKD, it has been widely considered that alleviating valve calcification can partially reduce cardiovascular events in patients with CKD. Cardiovascular disease (CVD) associated with aortic arch calcification can be detected in chest X-radiographs in the general population. ${ }^{12}$ A chronic renal insufficiency cohort study revealed that $\mathrm{CKD}$ patients have a $29 \%$ higher risk of heart failure history and developing ESRD, which is independently associated with the $50 \%$ decrease in GFR, when compared to non-CKD patients. ${ }^{13}$ Therefore, the prediction and prevention of heart failure are critical for CKD patients. Arterial calcification is commonly used for cardiovascular risk assessment. ${ }^{14}$ The severity of arterial calcification is closely correlated to the incidence and mortality of cardiovascular events.

For patients with maintenance hemodialysis, complications of cardiac valve calcification are common, and would increase the risk of death in hemodialysis patients. ${ }^{14}$ Therefore, it is important to understand the independent risk factors of cardiac valve calcification in hemodialysis patients. The prevention and treatment of many complications are very intractable when CKD patients develop to ESRD and require hemodialysis. ${ }^{15}$ Therefore, there is a need to identify independent risk factors for cardiac valve calcification in CKD patients as early as possible for early intervention, in order to improve the prognosis of CKD patients.

Fibroblast growth factor 23 (FGF23) is a member of the fibroblast growth factor (FGF) family. FGF23 is an endocrine-functional protein synthesized by osteocytes and osteoblasts. ${ }^{16}$ FGF23 has been proved to play an important role in the bone-kidney-parathyroid axis. ${ }^{16}$ This is regulated by blood phosphorus, active vitamin D, parathyroid hormone and Klotho, which are involved in maintaining the homeostasis of mineral metabolism in the body. ${ }^{17}$ FGF23 exerts its biological effects by binding to its receptor, which relies on the synergy of the Klotho protein. ${ }^{17}$ The Klotho gene has biological functions, such as anti-renal fibrosis, the regulation of calcium and phosphorus metabolism, and the participation of Wnt signal transduction. ${ }^{17}$ However, the causal relationship between FGF23 and valve calcification remains unknown. ${ }^{17}$ It remains controversial whether the elevation of FGF23 promotes valve calcification, or whether this is only a phenomenon in the whole process of mineral metabolism disorder associated with coronary calcification. ${ }^{17}$ FGF23 may be a novel treatment strategy to interrupt the potential $\mathrm{CV}$ risk associated with CKD. ${ }^{17}$

In order to further investigate the clinical value of Klotho and FGF23 in the cardiac valve calcification in patients with CKD, the present study intends to study the following aspects: (1) the relationship between the clinical data and CKD stages of subjects; (2) the influencing factor analysis of serum FGF23 expression in CKD patients; (3) the influencing factor analysis of serum Klotho expression in CKD patients; (4) the index analysis of CKD patients with cardiac valve calcification and CKD patients without cardiac valve calcification; (5) the risk factor analysis of cardiac valve calcification in CKD patients.

\section{Patients and Methods Research Objects}

Patients with CKD, who were treated in the Department of Renal Medicine of our hospital from April 1, 2016 to April 1, 2019, were enrolled in the present study as the main research subjects. According to the stages of CKD, these patients were divided into three groups: CKD2-3 group, CKD4 group and CKD5 group. Patients in each group were assessed by ultrasound for cardiac valve calcification. Enzyme-linked immunosorbent assay (ELISA) was used to detect the FGF23 (article No.: YB-FGF23HU, batch No.: E201907151347, Shanghai Yubo Biotechnology Co., Ltd) and serum Klotho (Article No.: YB-KL-HU, Batch No.: E201907151145, Shanghai Yubo Biotechnology Co., Ltd) expression levels. Logistic regression was used to analyze the independent risk factors for cardiac valve calcification. The present study was consistent with the Declaration of Helsinki of the World Medical Association. This study was conducted with approval from the Ethics Committee of the Second Affiliated Hospital of Nanchang University. All included patients provided a signed informed consent.

\section{Inclusion and Exclusion Criteria}

In this study, CKD patients who were admitted to the Department of Nephrology of our hospital on April 1, 2016 and solstice on April 1, 2019 were selected as the main research subjects. Patients were required to meet the relevant inclusion criteria and sign the informed consent. The sample size of the three groups of patients was 
designed to be the same, and not all eligible patients were included in the study.

The staging criteria and study grouping of patients with CKD included in the study were as follows: GFR 30-89 was stage 2 3 CKD; GFR 15-29 was the CKD4 group; GFR $<15$ was defined as CKD stage 5 group. The basic disease and drug treatment of the patients were routine treatment, and no intervention measures were taken in this study.

Inclusion criteria: (1) CKD history of more than one year; (2) >16 years old; (3) stage CKD2-5 patients; (4) patients who provided a signed informed consent; (5) patients with complete clinical data.

Exclusion criteria: (1) patients with acute renal failure; (2) patients with severe heart, liver and lung dysfunction and severe malnutrition; (3) patients with malignant tumors or other uncontrolled active infections, inflammation, and other diseases; (4) pregnant or lactating women; (5) patients with severe immune system diseases that are presently not in a stable state.

\section{Methods}

The CKD diagnosis was based on the diagnostic criteria of the K/DOQI Guidelines. The CKD staging criteria and study groups are presented, as follows: GFR $\geq 90$ is on the stage of CKD1, which is not included in the subgroup of the study; GFR 30-89 is on the stage of CKD2-3; GFR $15-29$ is on the stage of CKD4; GFR $<15$ is on the stage of CKD5.

Assessment of cardiac valve calcification: This was completed by full-time doctors through color Doppler ultrasound in our hospital. The aortic valve, mitral valve and valve ring of CKD patients were measured by echocardiography (frequency of the ultrasonic probe: 8-10 $\mathrm{MHz}$ ), and the cardiac valve calcification was evaluated. The ultrasound diagnosis of cardiac valve calcification was based on one or more massive or punctate hyperechoic masses, with a diameter of $\geq 1 \mathrm{~mm}$ on the heart valve or valve ring. All CKD patients were divided into two groups, according to the echocardiography: cardiac valve calcification group and non-cardiac valve calcification group.

\section{Clinical Index Test Methods}

After obtaining an informed consent, all CKD patients began fasting for food and beverages at 20:00 p.m. of the day before drawing blood. Venous blood was collected on an empty stomach for the detection of hemoglobin, albumin, blood urea, serum creatinine, blood uric acid, serum phosphorus, serum magnesium, osteocalcin, whole PTH, 25(OH) $\mathrm{VD}_{3}$ and osseous ALP. At the same time, blood samples were collected and tested for FGF23 and Klotho. After centrifuging and repacking, the samples were stored in the Biological Sample Library of our hospital. After all samples of CKD patients were collected, these were uniformly tested. Hemoglobin, albumin, blood urea, serum creatinine, blood uric acid, serum phosphorus, serum magnesium, osteocalcin, whole PTH, $1.25(\mathrm{OH})_{2}$ $\mathrm{VD}_{3}$, bone ALP and other biochemical indicators were uniformly detected by the laboratory of our hospital. The serum levels of FGF23 and Klotho was detected by ELISA, and the specific operation methods were strictly in accordance with the FGF23 and Klotho ELISA test kits.

\section{GFR Examination}

All CKD patients were examined with ${ }^{99} \mathrm{Tcm}$-DTPA renal dynamic imaging + glomerular filtration rate in the Nuclear Medicine Department of our hospital. The GFR of the lobulated kidney was measured. Total GFR $=$ GFR of the left lobulated kidney + GFR of the right lobulated kidney. The testing instrument used was the Infinia VC Hawkeye 4 dual-probe single-photon emission computed tomography (GE, USA). During the examination, the patient was instructed to take the recumbent position. The probe was close to the renal area of the waist, and the detection field included the kidney area. After a boluslike injection with ${ }^{99} \mathrm{Tcm}-\mathrm{DTPA}$, the dynamic images were immediately collected, the renogram was drawn, and the GFR of the lobulated kidney was calculated.

\section{Statistical Analysis}

The case data included in the study were collected, and a database of CKD patients was established. All relevant data were statistically analyzed using the SPSS 20.0 software, and the measurement data were expressed by mean \pm standard deviation $(\bar{x} \pm s)$. The enumeration data were expressed in percentage (\%). If the measurement data complied with the normal distribution, one-way analysis of variance (ANOVA) was used for comparisons among groups. Pearson's correlation analysis was used to analyze the influencing factors of FGF23 and Klotho. If the measurement data did not conform to the normal distribution, rank sum test was used for pairwise comparisons among groups. Indexes with statistical significance in the one-way ANOVA were included in the binary logistic regression analysis, and the cardiac valve calcification 
was taken as the dependent variable to investigate the independent risk factors that affect the cardiac valve calcification. Counting data were analyzed using chi-square test. $P<0.05$ was considered statistically significant.

\section{Results}

\section{The Relationship Between Gender, Age and the CKD Stages of Patients}

A total of $180 \mathrm{CKD}$ patients were enrolled in the present study, with an average age of $58 \pm 14$ years old. There were 113 males, accounting for $63 \%$, and 67 females, accounting for $37 \%$. According to the level of GFR, CKD patients were divided into three groups: $\mathrm{CKD} 2-3$ group $(n=60)$, with an average age of $54 \pm 13$ years old, including 38 males and 22 females; CKD4 group ( $n=60$ ), with an average age of $62 \pm 15$ years old, including 41 males and 19 females; CKD5 group $(n=60)$, with an average age of $58 \pm 15$ years old, including 34 males and 26 females. The analysis results show that the difference in age comparison was not statistically significant $(P=0.265)$ among patients of the different CKD groups. The difference in gender comparison was not statistically significant $(P=0.419)$ among patients of all groups included in the present study (Table 1).

\section{The Relationship Between Nutritional Indicators and the CKD Stages}

The present study examined the hemoglobin and albumin of CKD patients to assess the nutritional status of CKD patients. The results revealed that the hemoglobin was higher in CKD23 patients $(102.55 \pm 23.82 \mathrm{~g} / \mathrm{L})$ than in CKD4 patients $(86.68$ $\pm 17.33 \mathrm{~g} / \mathrm{L})$ and CKD5 patients $(90.52 \pm 23.36 \mathrm{~g} / \mathrm{L})$, and the difference was statistically significant $(P<0.05)$. The hemoglobin was higher in CKD5 patients $(90.52 \pm 23.36 \mathrm{~g} / \mathrm{L})$ than in CKD4 patients $(86.6 \pm 17.33 \mathrm{~g} / \mathrm{L})$, and the difference was statistically significant $(P<0.05)$. The albumin was lower in CKD2-3 patients $(32.63 \pm 7.20 \mathrm{~g} / \mathrm{L})$ than in CKD5 patients $(36.61 \pm 4.37 \mathrm{~g} / \mathrm{L})$, and the difference was statistically significant $(P<0.05)$. The albumin was higher in CKD5 patients $(36.61 \pm 4.37 \mathrm{~g} / \mathrm{L})$ than in CKD4 patients $(33.57 \pm 5.34 \mathrm{~g} / \mathrm{L})$, and the difference was statistically significant $(P<0.05)$ (Table 1).

\section{The Relationship Between Calcium and Phosphorus Metabolism Indexes and the CKD Stages}

The subjects included in the study were tested for calcium and phosphorus, and corrected for the serum calcium of
CKD patients with albumin $<40 \mathrm{~g} / \mathrm{L}$. These results revealed that the serum calcium was higher in CKD2-3 patients $(2.38 \pm 0.10 \mathrm{mmol} / \mathrm{L})$ than in CKD4 patients $(2.23$ $\pm 0.12 \mathrm{mmol} / \mathrm{L})$ and CKD5 patients $(2.06 \pm 0.15 \mathrm{mmol} / \mathrm{L})$, and the difference was statistically significant $(P<0.05)$. The serum calcium was higher in CKD4 patients $(2.23$ $\pm 0.12 \mathrm{mmol} / \mathrm{L})$ than in CKD5 patients $(2.06 \pm 0.15 \mathrm{mmol} /$ $\mathrm{L})$, and the difference was statistically significant $(P<0.05)$ (Table 1 and Figure 1).

\section{The Relationship Between Bone Metabolism Indicators and the CKD Stages}

The bone metabolism-related indexes of the study subjects tested in the present study included osteocalcin, PTH, serum 25-hydroxyvitamin D, and osseous ALP. The results revealed that the serum levels of osteocalcin, PTH and osseous ALP increased along with the decrease in GFR, and the difference in all indexes was statistically significant $(P<0.05)$ between CKD2-3 and CKD4, between CKD2-3 and CKD5, and between CKD4 and CKD5. The difference in the serum levels of serum 25-hydroxyvitamin D was not statistically significant between CKD2-3 (18.17 $\pm 10.22 \mathrm{ng} / \mathrm{mL})$ and CKD4 $(20.55 \pm 16.03 \mathrm{ng} / \mathrm{mL})$, while the difference was statistically significant $(P<0.05)$ between CKD2-3 (18.17 $\pm 10.22 \mathrm{ng} / \mathrm{mL})$ and CKD5 $(25.12 \pm 14.93 \mathrm{ng} / \mathrm{mL})$. The difference was not statistically significant between CKD4 $(20.55 \pm 16.03 \mathrm{ng} / \mathrm{mL})$ and CKD5 $(25.12 \pm 14.93 \mathrm{ng} / \mathrm{mL})$ (Table 1 and Figure 2).

\section{The Relationship Between FGF23, Klotho Expression and the CKD Stages}

The ELISA results indicated that the serum levels of FGF23 and Klotho increased along with the decrease in GFR. The serum levels of FGF23 was lower in CKD2-3 patients (494.60 $\pm 64.85 \mathrm{pg} / \mathrm{mL})$ than in CKD4 patients $(721.17$ $\pm 92.05 \mathrm{pg} / \mathrm{mL})$ and CKD5 patients $(1039.43 \pm 213.83 \mathrm{pg} /$ $\mathrm{mL}$ ). The serum levels of FGF23 was lower in CKD4 patients $(721.17 \pm 92.05 \mathrm{pg} / \mathrm{mL})$ than in CKD5 patients $(1039.43$ $\pm 213.83 \mathrm{pg} / \mathrm{mL}$ ). The difference was statistically significant $(P<0.05)$ among the subgroups. The serum levels of Klotho was higher in CKD2-3 patients $(159.05 \pm 27.53 \mathrm{U} / \mathrm{L})$ than in CKD4 patients (127.58 $\pm 16.85 \mathrm{U} / \mathrm{L})$ and CKD5 patients $(87.06 \pm 15.14 \mathrm{U} / \mathrm{L})$. The serum levels of Klotho was higher in CKD4 patients $(127.58 \pm 16.85 \mathrm{U} / \mathrm{L})$ than in CKD5 patients $(87.06 \pm 15.14 \mathrm{U} / \mathrm{L})$. The difference was statistically significant $(P<0.05)$ among the subgroups (Table 1 and Figure 3). 
Table I The Relationship Between Clinical Data and the CKD Stages of Patients

\begin{tabular}{|c|c|c|c|c|}
\hline CKD Stages & CKD 2-3 & CKD 4 & CKD 5 & $\mathbf{P}$ \\
\hline Cases(n) & 60 & 60 & 60 & \\
\hline Gender(M/F) & $38 / 22$ & $41 / 19$ & $34 / 26$ & 0.419 \\
\hline Average age(Y) & $54 \pm 13$ & $56 \pm 12$ & $58 \pm 15$ & 0.265 \\
\hline Hemoglobin(g/L) & $102.55 \pm 23.82$ & $86.68 \pm 17.33$ & $90.52 \pm 23.36$ & 0.000 \\
\hline Albumin(g/L) & $32.63 \pm 7.20$ & $33.57 \pm 5.34$ & $36.61 \pm 4.37$ & 0.001 \\
\hline Urea(mmol/L) & $10.20 \pm 5.19$ & $|8.4| \pm 8.15$ & $24.08 \pm 10.11$ & 0.000 \\
\hline Serum creatinine(umol/L) & $230.61 \pm 185.52$ & $491.72 \pm 215.32$ & $845.09 \pm 334.18$ & 0.000 \\
\hline $\mathrm{GFR}(\mathrm{mL} / \mathrm{min})$ & $47.04 \pm 14.00$ & $21.84 \pm 5.01$ & $10.14 \pm 2.13$ & 0.000 \\
\hline Blood uric acid(umol/L) & $360.67 \pm 100.35$ & $440.81 \pm 169.23$ & $574.33 \pm 95.34$ & 0.000 \\
\hline B2 microglobulin(mg/L) & $8.57 \pm 5.4 I$ & $13.28 \pm 4.03$ & $16.52 \pm 3.47$ & 0.000 \\
\hline Correction of calcium(mmol/L) & $2.38 \pm 0.10$ & $2.23 \pm 0.12$ & $2.06 \pm 0.15$ & 0.000 \\
\hline Blood phosphorus(mmol/L) & $1.22 \pm 0.27$ & $1.59 \pm 0.42$ & $1.92 \pm 0.56$ & 0.000 \\
\hline Blood magnesium(mmol/L) & $0.91 \pm 0.15$ & $0.95 \pm 0.16$ & $1.00 \pm 0.18$ & 0.015 \\
\hline Osteocalcin(ng/mL) & $47.99 \pm 35.82$ & $104.43 \pm 73.22$ & $197.32 \pm 78.88$ & 0.000 \\
\hline $\mathrm{PTH}(\mathrm{pg} / \mathrm{mL})$ & $|3| .|3 \pm 90.4|$ & $254.01 \pm 184.68$ & $392.40 \pm 233.88$ & 0.005 \\
\hline Serum 25-hydroxyvitamin $D(n g / m L)$ & $18.17 \pm 10.22$ & $20.55 \pm 16.03$ & $25.12 \pm 14.93$ & 0.023 \\
\hline Osseous ALP(U/L) & $77.27 \pm 1 \mid .56$ & $81.33 \pm 15.15$ & $86.36 \pm 18.18$ & 0.005 \\
\hline Klotho(U/L) & $159.05 \pm 27.53$ & $127.58 \pm 16.85$ & $87.06 \pm 15.14$ & 0.000 \\
\hline FGF23(pg/mL) & $494.60 \pm 64.85$ & $721.17 \pm 92.05$ & $1039.43 \pm 213.83$ & 0.000 \\
\hline
\end{tabular}

\section{Influencing Factor Analysis of Serum FGF23 Expression in CKD Patients}

Pearson's correlation analysis was carried out between serum FGF23 and age, Klotho, osteocalcin, PTH, serum 25hydroxyvitamin D, osseous ALP, serum phosphorus, corrected calcium, $\beta-2$ microglobulin, GFR, albumin, blood uric acid, serum creatinine and serum magnesium in CKD patients, and the results revealed that FGF23 was negatively correlated to Klotho, corrected calcium and GFR (the $\mathrm{r}$ values were $-0.836,-0.579$ and -0.718 , respectively). FGF23 was positively correlated with osteocalcin, PTH, serum 25-hydroxyvitamin D, osseous ALP, serum phosphorus, $\beta$-2-microglobulin, albumin, blood uric acid, serum creatinine, serum magnesium and age (the $r$ values were $0.563,0.348,0.207,0.213,0.462,0.495,0.249,0.496$, $0.580,0.224$ and 0.179 , respectively) (Table 2).

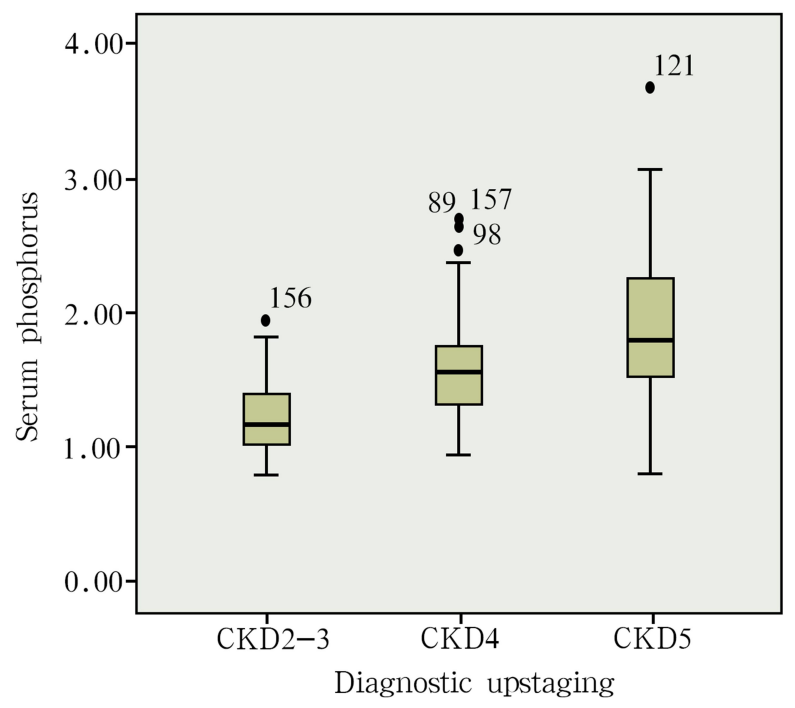

Figure I The relationship between calcium and phosphorus metabolism indexes and the CKD stages. 

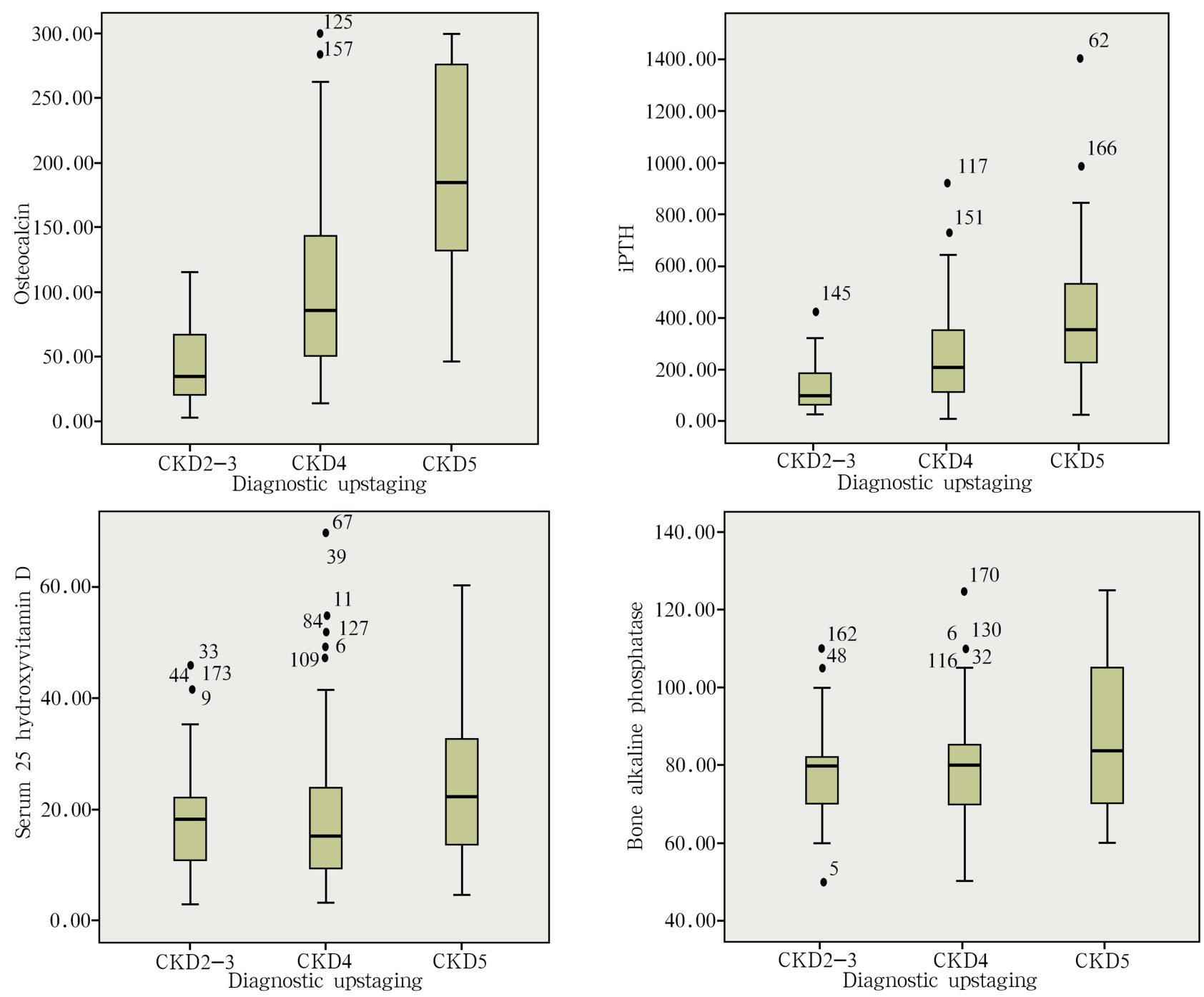

Figure 2 The relationship between bone metabolism indicators and the CKD stages.

\section{Influencing Factor Analysis of Serum Klotho Expression in CKD Patients}

Pearson's correlation analysis was carried out between serum Klotho and age, FGF23, osteocalcin, PTH, serum 25hydroxyvitamin D, osseous ALP, serum phosphorus, corrected calcium, $\beta$-2-microglobulin, GFR, albumin, blood uric acid, serum creatinine and serum magnesium in CKD patients, and the results revealed that Klotho is negatively correlated with FGF23, osteocalcin, PTH, osseous ALP, serum phosphorus, $\beta$-2-microglobulin, albumin, blood uric acid and serum creatinine (the $\mathrm{r}$ values were $-0.836,-0.581$, $-0.378,-0.181,-0.487,-0.486,-0.186,-0.493$ and -0.573 , respectively). Klotho was positively correlated with corrected calcium and GFR (the $r$ values were 0.562 and 0.721, respectively). However, the serum levels of Klotho was not correlated with age, serum magnesium, serum 25hydroxyvitamin D and the other indexes (Table 3).

\section{Index Analysis of CKD Patients with Cardiac Valve Calcification and CKD Patients without Cardiac Valve} Calcification

CKD patients were divided into two groups, according to the color Doppler ultrasound: cardiac valve calcification group and non-cardiac valve calcification group. A total of 180 CKD patients were enrolled in the present study. Among these patients, 41 patients were included in the cardiac valve calcification group, accounting for $22.7 \%$, and including four patients with stage CKD2-3 cardiac valve calcification, 12 patients with stage CKD4 cardiac 


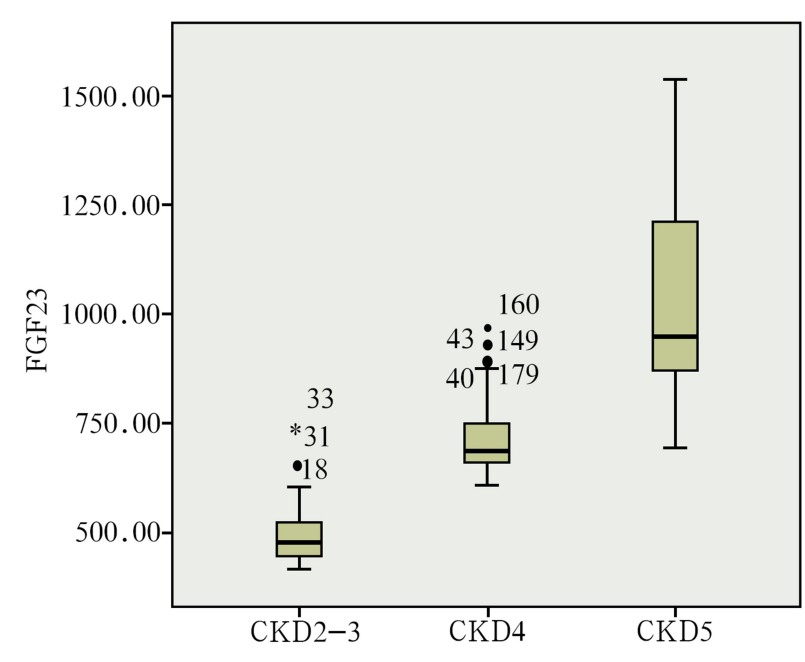

Diagnostic upstaging

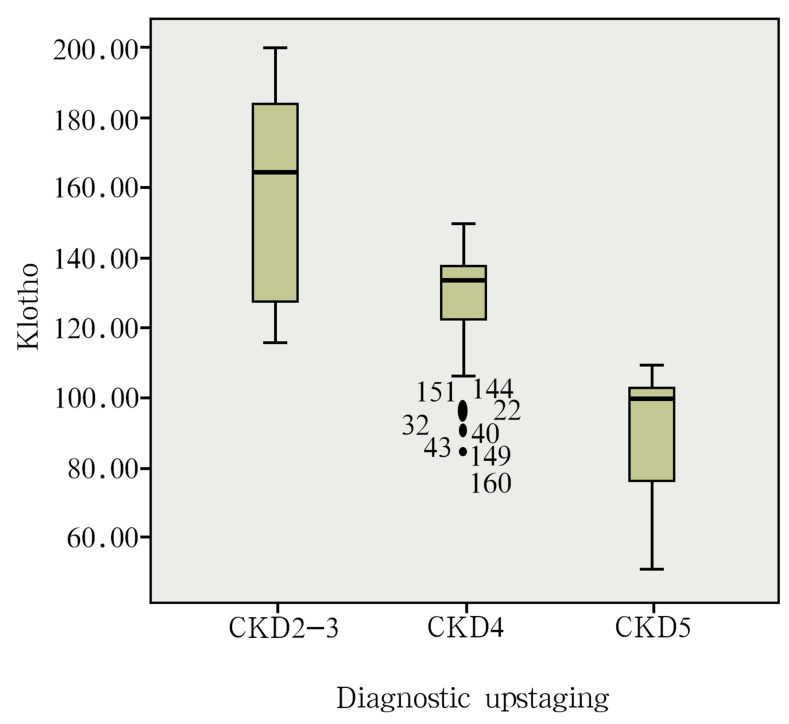

Figure 3 The relationship between FGF23, Klotho expression and the CKD stages.

valve calcification, and 25 patients with stage CKD5 cardiac valve calcification, while 139 patients were included in the non-cardiac valve calcification group, accounting for $77.3 \%$. The analysis results revealed that the difference in FGF23, Klotho, osteocalcin, serum phosphorus, $\beta$-2-microglobulin, corrected calcium, age, albumin, urea, serum creatinine, GFR, blood uric acid, serum magnesium and other indexes was statistically significant (all, $P<0.05$ ) between the CKD group with cardiac valve calcification and the CKD group with noncardiac valve calcification (Table 4).

Table 2 Influencing Factor Analysis of Serum FGF23 Expression in CKD Patients

\begin{tabular}{|l|l|l|}
\hline Index & r & P \\
\hline Klotho(U/L) & -0.836 & $<0.05$ \\
Osteocalcin(ng/mL) & 0.563 & $<0.05$ \\
PTH(pg/mL) & 0.348 & $<0.05$ \\
Serum 25-hydroxyvitamin D(ng/mL) & 0.207 & 0.005 \\
Osseous ALP(U/L) & 0.213 & 0.004 \\
Blood phosphorus(mmol/L) & 0.462 & $<0.05$ \\
Correction of calcium(mmol/L) & -0.579 & $<0.05$ \\
B2 microglobulin(mg/L) & 0.495 & $<0.05$ \\
GFR(mL/min) & -0.718 & $<0.05$ \\
Albumin(g/L) & 0.249 & 0.001 \\
Age(Y) & 0.179 & 0.016 \\
Blood uric acid(umol/L) & 0.496 & $<0.05$ \\
Serum creatinine(umol/L) & 0.58 & $<0.05$ \\
Blood magnesium(mmol/L) & 0.224 & 0.003 \\
\hline
\end{tabular}

\section{Risk Factor Analysis of Cardiac Valve Calcification in CKD Patients}

For the purpose of understanding the risk factors of cardiac valve calcification in CKD patients, two Logistic regression analysis were carried out to determine whether these patients were accompanied by cardiac valve calcification as dependent variables, while FGF23, Klotho, osteocalcin, serum phosphorus, $\beta$-2-microspheres, corrected calcium, age, albumin, urea, serum creatinine, GFR, blood uric acid and serum magnesium were the independent variables. These results reveal that GFR, serum creatinine, FGF23 and Klotho are independent risk factors for cardiac valve calcification in CKD patients (Table 5).

\section{Discussion}

Malnutrition is also a risk factor for cardiovascular events in CKD patients. The present study on the relationship among hemoglobin, albumin and CKD stages in CKD patients revealed that the hemoglobin was higher in CKD5 patients than in CKD4 patients. The further retrospective analysis of patient medical records revealed that the higher hemoglobin may be correlated to the use of recombinant human erythropoietin in CKD5 patients, who are mainly inpatients, while CKD4 patients were mainly outpatients. Due to the poor compliance of recombinant human erythropoietin under subcutaneous administration, this may lead to the poor improvement of hemoglobin indexes. Furthermore, the albumin was lower in CKD2-3 
Table 3 Influencing Factor Analysis of Serum Klotho Expression in CKD Patients

\begin{tabular}{|l|l|l|}
\hline Index & r & P \\
\hline FGF23(pg/mL) & -0.836 & $<0.05$ \\
Osteocalcin(ng/mL) & -0.581 & $<0.05$ \\
PTH(pg/mL) & -0.378 & $<0.05$ \\
Osseous ALP(U/L) & -0.181 & 0.015 \\
Blood phosphorus(mmol/L) & -0.487 & $<0.05$ \\
Correction of calcium(mmol/L) & 0.562 & $<0.05$ \\
B2 microglobulin(mg/L) & -0.486 & $<0.05$ \\
GFR(mL/min) & 0.721 & $<0.05$ \\
Albumin(g/L) & -0.186 & 0.012 \\
Blood uric acid(umol/L) & -0.493 & $<0.05$ \\
Serum creatinine(umol/L) & -0.573 & $<0.05$ \\
\hline
\end{tabular}

patients than in CKD5 patients. The cause analysis revealed that the majority of CKD2-3 patients suffered from nephrotic syndrome. Furthermore, some of them were not controlled. Therefore, proteinuria still existed, resulting in hypoproteinemia. The protein intake of CKD4 patients was limited due to the low protein diet, while that of CKD5 patients was partially broadened due to the fact that some of them started the hemodialysis treatment. Therefore, the albumin was higher in CKD5 patients than in CKD4 patients in the present study.

The risk factors for cardiac valve calcification include valvular dysfunction, hypertension, cardiac ischemia, conduction disorders of ECG activity, cardiac dysfunction and infective endocarditis. Among these CKD patients, the incidence of cardiac valve calcification was presently lacking in large sample studies. Previous studies have revealed that the incidence of cardiac valve calcification is more than $20 \%{ }^{15,16}$ The incidence of cardiac valve calcification was $22.7 \%$ among the 180 patients with CKD2-5 in the present study. It was slightly lower, when compared to that of CKD patients as research subjects. This may be associated to the increase in sample size in the present study.

FGF23 is a key factor found in recent years to regulate calcium and phosphorus, which is mainly synthesized and secreted by osteoblasts. ${ }^{18}$ This plays a physiological role in combination with its corresponding complex receptors. The membranous Klotho protein is the main component of its specific complex receptors. ${ }^{18}$ The excretion of serum phosphorus is affected by the decrease in GFR in the early stage of CKD. ${ }^{18}$ With the further decrease in GFR, the level of FGF23 increased. ${ }^{19}$ However, since the urine excretion of most CKD patients decreased along with the decrease in GFR, the increase in FGF23 failed to promote the excretion of more urine phosphorus, resulting in abnormal calcium and phosphorus metabolism in patients. ${ }^{19}$ The abnormal calcium and phosphorus metabolism stimulates the further increase of FGF $23 .{ }^{19}$ Furthermore, FGF 23 is mainly metabolized by the kidneys, and its molecular weight is large, making it not easy to be removed by dialysis. ${ }^{19}$ Hence, the serum FGF23 level of patients can reach up to 1000 times of normal people. ${ }^{17}$ The results of the present study reveal that the serum levels of FGF23 increases with the decrease in GFR. The influencing factors of FGF23 include osteocalcin, PTH, serum 25-hydroxyvitamin D, osseous ALP, serum phosphorus, $\beta-2$ microglobulin, albumin, blood uric acid, serum creatinine, serum magnesium and age. These factors are positively correlated with the serum levels of FGF23, including strong correlation coefficients, such as osteocalcin, $\beta$-2-microglobulin and serum creatinine,

Table 4 Index Analysis of CKD Patients with Cardiac Valve Calcification and CKD Patients without Cardiac Valve Calcification

\begin{tabular}{|l|l|l|l|}
\hline Index & Non-Cardiac Valve Calcification Group & Cardiac Valve Calcification Group & P \\
\hline FGF23(pg/mL) & $657.7 \pm 15.69$ & $1070.54 \pm 37.59$ & $<0.05$ \\
Klotho(U/L) & $136.1 I \pm 2.66$ & $85.44 \pm 2.99$ & $<0.05$ \\
Osteocalcin(ng/mL) & $105.63 \pm 7.58$ & $153.7 \pm 12.76$ & 0.002 \\
Blood phosphorus(mmol/L) & $1.54 \pm 0.04$ & $1.72 \pm 0.09$ & 0.04 \\
B2 microglobulin(mg/L) & $12.27 \pm 0.47$ & $14.57 \pm 0.73$ & 0.017 \\
Correction of calcium(mmol/L) & $2.25 \pm 0.02$ & $2.17 \pm 0.03$ & 0.021 \\
Age (Y) & $55.37 \pm 1.19$ & $60.37 \pm 1.84$ & 0.039 \\
Albumin(g/L) & $33.89 \pm 0.52$ & $35.6 \pm 0.8$ & 0.108 \\
Urea(mmol/L) & $16.72 \pm 0.78$ & $20.43 \pm 1.79$ & 0.034 \\
Serum creatinine(umol/L) & $488.5 I \pm 29.46$ & $637.62 \pm 57.45$ & 0.018 \\
GFR(mL/min) & $29.25 \pm 1.56$ & $16.51 \pm 1.63$ & $<0.05$ \\
Blood uric acid(umol/L) & $438.8 \pm 12.46$ & $525.79 \pm 24.7$ & 0.001 \\
Blood magnesium(mmol/L) & $0.95 \pm 0.01$ & $0.98 \pm 0.03$ & $0.31 \mathrm{I}$ \\
\hline
\end{tabular}


Table 5 Risk Factor Analysis of Cardiac Valve Calcification in CKD Patients

\begin{tabular}{|l|c|l|l|l|l|}
\hline Index & B & SE & Wald & OR(95\% CI) \\
\hline GFR & 0.082 & 0.028 & 8.513 & $1.085(I .027-1.147)$ & P \\
Serum creatinine & -0.004 & 0.001 & 10.015 & $0.996(0.993-0.998)$ & 0.004 \\
FGF23 & 0.012 & 0.004 & 10.500 & $1.012(1.005-1.019)$ & 0.002 \\
Klotho & -0.072 & 0.024 & 9.428 & $0.930(0.888-0.974)$ & 0.001 \\
\hline
\end{tabular}

suggesting that adequate dialysis, especially highthroughput hemodialysis, may be effective in reducing FGF23 expression in clinical maintenance hemodialysis patients, thereby reducing cardiovascular events and improving the quality of life of hemodialysis patients. This provides a theoretical basis for adequate hemodialysis and high-quality hemodialysis in clinic.

The high serum levels of FGF23 can increase the risks of cardiac valve calcification, cardiac insufficiency, cardiomyocyte hypertrophy and arteriosclerosis in CKD patients. ${ }^{18}$ The results of the present study reveal that the level of FGF23 significantly increases in CKD patients with cardiac valve calcification. The binary logistic regression analysis suggests that FGF23 is an independent risk factor for patients with cardiac valve calcification.

Klotho is mainly expressed in renal proximal convoluted tubules and parathyroid glands, including membranous Klotho protein and soluble Klotho protein. ${ }^{20}$ The soluble Klotho protein is formed by the extracellular segment of membranous Klotho protein falling off into the blood. $^{20}$ Membranous Klotho protein plays a biological role by relying on FGF23, while soluble Klotho protein does not rely on the FGF23 pathway to play a biological role in regulating ion channels and transporters. ${ }^{20}$ The present study revealed that the serum levels of Klotho protein is negatively correlated with FGF23, osteocalcin, PTH, osseous ALP, serum phosphorus, $\beta$-2-microglobulin, albumin, blood uric acid and serum creatinine. The serum levels of Klotho protein is positively correlated with the corrected calcium, GFR and other indexes. This may be due to the decrease in the number of cells that can express Klotho protein, the decrease in the level of GFR protein due to the atrophy of glomeruli, and the decrease in the function of renal tubules caused the decrease in Klotho protein. ${ }^{20}$ The results of the present study show that the level of Klotho protein is not associated with age, serum magnesium, serum 25-hydroxyvitamin D and other indexes, but not excluding its correlation to the small sample size.
There may be other mechanisms. However, there is no relevant research at present.

Diabetes is an important risk factor for cardiac valve

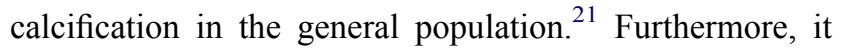
has been confirmed that a history of diabetes is a risk factor for cardiovascular events in maintenance hemodialysis patients. ${ }^{21}$ The occurrence of cardiac valve calcification in CKD patients with diabetes was not statistically significant, when compared to CKD patients without diabetes. The logistic regression analysis suggests that a history of diabetes is not an independent risk factor for cardiac valve calcification. Hence, this needs to be further studied with a larger sample size.

The study on the risk factors of cardiac valve calcification revealed that the GFR, serum creatinine, FGF23 and Klotho of CKD patients are independent risk factors that affect cardiac valve calcification in CKD patients. Therefore, early intervention is very important for CKD patients to prevent CVD complications through delaying the progress of renal dysfunction, and reducing the risk factors of GFR decline, such as effective control of blood pressure, blood sugar and blood lipids, and avoiding the use of nephrotoxic drugs.

There were still limitations in the present study. First, the present study was a case control study, rather than a randomized controlled trial. Therefore, there is still a certain risk of bias. Second, the present study is a singlecenter clinical study, and the sample size was small. Thus, it is still necessary to further increase the sample size and carry out multicenter clinical studies. Finally, the results of the present study revealed that a history of diabetes is not an independent risk factor for cardiac valve calcification. Therefore, the correlation between a history of diabetes and cardiac valve calcification still needs to be further investigated.

\section{Conclusion}

With the decrease in GFR in CKD patients, FGF23 expression increases and Klotho expression decreases. The serum levels of FGF23 and Klotho is negatively correlated with 
the FGF23 and Klotho levels in CKD patients. GFR, serum creatinine, FGF23 and Klotho were the independent risk factors for cardiac valve calcification in CKD patients.

\section{Acknowledgments}

We would like to acknowledge the hard and dedicated work of all the staff that implemented the intervention and evaluation components of the study.

\section{Funding}

This study was funded by Key research and development projects of Jiangxi science and Technology Department (Grant No: 20171BBG70071). The funding body had no role in the design of the study and collection, analysis, and interpretation of data and in writing the manuscript.

\section{Disclosure}

The authors declare that they have no conflicts of interest for this work.

\section{References}

1. Bundy JD, Chen J, Yang W, et al. Risk factors for progression of coronary artery calcification in patients with chronic kidney disease: the CRIC study. Atherosclerosis. 2018;271:53-60. doi:10.1016/j. atherosclerosis.2018.02.009

2. Dong Y, Wang Z, Chen Z, et al. Comparison of visceral, body fat indices and anthropometric measures in relation to chronic kidney disease among Chinese adults from a large scale cross-sectional study. BMC Nephrol. 2018;19(1):40. doi:10.1186/s12882-018-0837-1

3. Bover J, Ureña $P$, Aguilar A, et al. Alkaline phosphatases in the complex chronic kidney disease-mineral and bone disorders. Calcif Tissue Int. 2018;103(6):1-14. doi:10.1007/s00223-018-0399-z

4. Tangri N, Stevens LA, Griffith J, et al. A predictive model for progression of chronic kidney disease to kidney failure. JAMA. 2011;305 (15):1553-1559. doi:10.1001/jama.2011.451

5. Gansevoort RT, Correa-Rotter R, Hemmelgarn BR, et al. Chronic kidney disease and cardiovascular risk: epidemiology, mechanisms, and prevention. Lancet. 2013;382(9889):339-352. doi:10.1016/ S0140-6736(13)60595-4

6. Kim HJ, Kang E, Oh YK, et al. The association between soluble klotho and cardiovascular parameters in chronic kidney disease: results from the KNOW-CKD study. BMC Nephrol. 2018;19(1):51. doi:10.1186/s12882-018-0851-3

7. Lamb EJ, Levey AS, Stevens PE. The Kidney Disease Improving Global Outcomes (KDIGO) guideline update for chronic kidney disease: evolution not revolution. Clin Chem. 2013;59(3):462-465. doi:10.1373/clinchem.2012.184259

International Journal of General Medicine

\section{Publish your work in this journal}

The International Journal of General Medicine is an international, peer-reviewed open-access journal that focuses on general and internal medicine, pathogenesis, epidemiology, diagnosis, monitoring and treatment protocols. The journal is characterized by the rapid reporting of reviews, original research and clinical studies
8. Oh J, Wunsch R, Turzer M, et al. Advanced coronary and carotid arteriopathy in young adults with childhood-onset chronic renal failure. Circulation. 2002;106(1):100-105. doi:10.1161/01. CIR.0000020222.63035.C0

9. Peeters MJ, van den Brand JA, van Zuilen AD, et al. Abdominal aortic calcification in patients with CKD. J Nephrol. 2017;30 (1):109-118. doi:10.1007/s40620-015-0260-7

10. Haydar AA, Covic A, Colhoun H, et al. Coronary artery calcification and aortic pulse wave velocity in chronic kidney disease patients. Kidney Int. 2004;65(5):1790-1794. doi:10.1111/j.15231755.2004.00581.x

11. De Vinuesa SG, Ortega M, Martinez P, et al. Subclinical peripheral arterial disease in patients with chronic kidney disease: prevalence and related risk factors. Kidney Int. 2005;67(s93):S44-S47. doi:10.1111/j.1523-1755.2005.09310.x

12. Iribarren C, Sidney S, Sternfeld B, et al. Calcification of the aortic arch: risk factors and association with coronary heart disease, stroke, and peripheral vascular disease. JAMA. 2000;283(21):2810-2815. doi:10.1001/jama.283.21.2810

13. Rahman M, Xie D, Feldman HI, et al. Association between chronic kidney disease progression and cardiovascular disease: results from the CRIC Study. Am J Nephrol. 2014;40(5):399-407. doi:10.1159/ 000368915

14. Levitzky YS, Cupples LA, Murabito JM, et al. Prediction of intermittent claudication, ischemic stroke, and other cardiovascular disease by detection of abdominal aortic calcific deposits by plain lumbar radiographs. Am J Cardiol. 2008;101(3):326-331. doi:10.1016/j.amjcard.2007.08.032

15. Yiu KH, Graaf FRD, Velzen JEV, et al. Different value of coronary calcium score to predict obstructive coronary artery disease in patients with and without moderate chronic kidney disease. Netherlands Heart J. 2013;21(7-8):347-353. doi:10.1007/s12471013-0409-8

16. P F S I, Daniel J, Moss SC, Goldman CK, Woods TC. Lightell. elevated serum bone morphogenetic protein 4 in patients with chronic kidney disease and coronary artery disease. J Cardiovasc Transl Res. 2013;6(2):232-238. doi:10.1007/s12265-012-9429-9

17. Andrukhova O, Slavic S, Smorodchenko A, et al. FGF23 regulates renal sodium handling and blood pressure. EMBO Mol Med. 2014;6 (6):744-759. doi:10.1002/emmm.201303716

18. Nitta K. Fibroblast growth factor 23 and cardiovascular disease in patients with chronic kidney disease. Renal Replacement Therapy. 2018;4(1):1-8. doi:10.1186/s41100-018-0172-9

19. Fukuda-Tatano S, Yamamoto H, Nakahashi O, et al. Regulation of $\alpha$ klotho expression by dietary phosphate during growth periods. Calcif Tissue Int. 2019;104(6):667-678. doi:10.1007/s00223-019-00525-0

20. Navarro-González JF, Sánchez-Niño MD, Donate-Correa J, et al. Effects of pentoxifylline on soluble Klotho concentrations and renal tubular cell expression in diabetic kidney disease. Diabetes Care. 2018;41(8):1817-1820. doi:10.2337/dc18-0078

21. Mantovani A, Pernigo M, Bergamini C, et al. Heart valve calcification in patients with type 2 diabetes and nonalcoholic fatty liver disease. Metabolism. 2015;64(8):879-887. doi:10.1016/j. metabol.2015.04.003

across all disease areas. The manuscript management system is completely online and includes a very quick and fair peer-review system, which is all easy to use. Visit http://www.dovepress.com/ testimonials.php to read real quotes from published authors. 